\title{
Experimental Investigation of Reflection Coefficient and Wigner's Reaction Matrix for Microwave Graphs
}

\author{
O. Hul, S. Bauch, M. Ławniczak and L. Sirko \\ Institute of Physics, Polish Academy of Sciences \\ al. Lotników 32/46, 02-668 Warszawa, Poland
}

We present the results of experimental study of the distribution $P(R)$ of the reflection coefficient $R$ and the distributions of Wigner's reaction $K$ matrix for irregular, tetrahedral microwave graphs (networks) in the presence of absorption. Our experimental results are in good agreement with the exact theoretical predictions of Savin et al.

PACS numbers: 05.45.Mt, 03.65.Nk

\section{Introduction}

Quantum graphs of connected one-dimensional wires were introduced by Pauling seven decades ago [1]. The same idea was used a decade later by Kuhn [2] to describe organic molecules by free electron models. Quantum graphs can be considered as idealizations of physical networks in the limit where the lengths of the wires greatly exceed their widths assuming that propagating waves remain in a single transverse mode. Among the systems modeled by quantum graphs are, e.g., electromagnetic optical waveguides [3, 4], mesoscopic systems [5, 6], quantum wires $[7,8]$ and excitation of fractons in fractal structures $[9,10]$. Recent works have shown that quantum graphs provide an excellent system for studying quantum chaos [11-21]. Quantum graphs with external leads (antennas) have been analyzed in detail in $[16,17]$. Quantum graphs with absorption, more realistic but more complicated systems, have been recently studied in [20,21].

In this paper we present the results of the experimental study of the distribution $P(R)$ of the reflection coefficient $R$ and the distributions of Wigner's reaction matrix [22] (also called the $K$ matrix [23]) for microwave networks that correspond to graphs with time reversal symmetry $(\beta=1$ symmetry class of random matrix theory [24]) in the presence of absorption. Our experimental results are compared with the exact theoretical predictions of Savin et al. [25]. 


\section{Theory}

In the case of systems coupled by a single-channel antenna, the $K$ matrix and the scattering matrix $S$ are related by

$$
S=\frac{1-\mathrm{i} K}{1+\mathrm{i} K} .
$$

The function $K=-\mathrm{i} Z$ and therefore the scattering matrix $S=(1-Z) /(1+Z)$ are directly connected with the impedance $Z$, which has been recently measured in a microwave cavity experiment $[26,27]$. The $S$ matrix can be parameterized as

$$
S=\sqrt{R} \mathrm{e}^{\mathrm{i} \theta},
$$

where $R$ is the reflection coefficient and $\theta$ is the phase.

After pioneering work of López et al. [28] came theoretical studies of the properties of statistical distributions of the $S$ matrix with direct processes and imperfect coupling [29-31]. In a recent experiment work [32] the distribution of the $S$ matrix for chaotic microwave cavities with absorption was investigated. The distribution $P(R)$ of the reflection coefficient $R$ in Eq. (2), at the beginning investigated theoretically in the strong absorption limit [33], has been recently known for any dimensionless absorption strength $\gamma=2 \pi \Gamma / \Delta$, where $\Gamma$ is the absorption width and $\Delta$ is the mean level spacing [25]. For systems with time reversal symmetry $(\beta=1)$ Méndez-Sánchez et al. [34] studied $P(R)$ experimentally, and Savin et al. [25] found an exact formula for $P(R)$. Beenakker and Brouwer [35] calculated $P(R)$ for systems violating time reversal symmetry $(\beta=2)$, in the case of a perfectly coupled, single-channel lead.

In our experiment we simulate quantum graphs with microwave networks. The analogy between them is based on the equivalence of the Schrödinger equation for the former to the telegraph equation for the latter [20].

A simple tetrahedral microwave graph consists of $N=4$ joints (vertices) and six coaxial cables (bonds). Each coaxial cable consists of an inner conductor with radius $r_{1}$ separated from a concentric outer conductor with inner radius $r_{2}$ by a homogeneous, non-magnetic material with dielectric constant $\varepsilon$. The fundamental TEM mode that propagates (the so-called Lecher wave) down to zero frequency exists because the cross-section of the cable is doubly connected [36, p. 253]. Below the frequency $\nu_{c} \simeq \frac{c}{\pi\left(r_{1}+r_{2}\right) \sqrt{\varepsilon}}[36]$, above which the other modes can propagate, the cable is single mode: only the TEM mode propagates. For SMA-RG-402 coaxial cables, which were used in the experiment, $r_{1}=0.05 \mathrm{~cm}, r_{2}=0.15 \mathrm{~cm}$, and $\varepsilon \simeq 2.08$ (teflon dielectric), single-mode propagation occurs below $32.9 \mathrm{GHz}$.

A graph with no absorption and no leads to the outside world is a closed system. The presence of absorption and/or leads creates an open system. We may vary absorption in the microwave graphs by adding one or more (coaxial) microwave attenuators, changing the length of cable(s) [20], or by changing the coupling to the outside world.

It is convenient to find the distribution $P(R)$ of the reflection coefficient $R$ and the distributions of imaginary and real parts of Wigner's reaction matrix $K$ 
using the impedance approach $[26,27]$. In this approach one can easily obtain the real and imaginary parts of the normalized impedance

$$
Z=\frac{\operatorname{Re} Z_{\mathrm{s}}+\mathrm{i}\left(\operatorname{Im} Z_{s}-\operatorname{Im} Z_{\mathrm{r}}\right)}{\operatorname{Re} Z_{\mathrm{r}}}
$$

of a chaotic microwave system, where $Z_{\mathrm{s}(\mathrm{r})}=Z_{0}\left(1+S_{\mathrm{s}(\mathrm{r})}\right) /\left(1-S_{\mathrm{s}(\mathrm{r})}\right)$ is the system (radiation) impedance expressed by the system (radiation) scattering matrix $S_{\mathrm{s}(\mathrm{r})}$ and $Z_{0}$ is the characteristic impedance of the transmission line. The radiation impedance $Z_{\mathrm{r}}$ is the impedance seen at the input of the coupling structure for the same coupling geometry, but with the vertices (walls) of the system removed to infinity. Wigner's reaction matrix $K$ can be expressed by the normalized impedance $K=-\mathrm{i} Z$ and the scattering matrix $S$ of a graph for the perfect coupling case (no direct processes present) can be extracted from the formula $S=(1-Z) /(1+Z)$. This interesting approach is especially useful in the studies of microwave systems in which both the system and radiation impedances are measurable.

\section{Results and discussion}

Figure 1 shows the experimental setup for measuring of the single-channel scattering matrix $S_{\mathrm{s}}$ of tetrahedral microwave graphs necessary for finding the system impedance $Z_{\mathrm{s}}$. We used a microwave vector network analyzer to measure the scattering matrix $S_{\mathrm{s}}$ in a frequency window of 3.5-7.5 GHz. At one of the four vertices of the microwave graph we used a 4 -joint connector to couple the graph to the vector network analyzer via a single-channel lead realized with a low-loss flexible microwave cable. The other three vertices were $T$-joints. Let us note that the microwave graph in Fig. 1 has a microwave attenuator in one of its bonds.

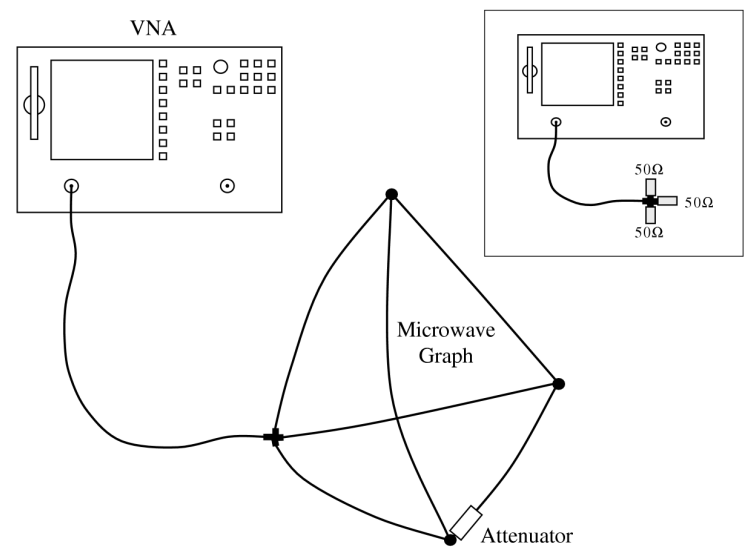

Fig. 1. The experimental setup used to measure the scattering matrix $S_{\mathrm{s}}$ of tetrahedral microwave graphs with absorption. Absorption in the graphs was varied by changing the attenuator. In the inset we show the setup used to measure the radiation scattering matrix $S_{\mathrm{r}}$. Instead of a graph three $50 \Omega$ loads were connected to the 4 -joint. 
The radiation impedance $Z_{\mathrm{r}}$ was found experimentally by measuring the scattering matrix $S_{\mathrm{r}}$ of the 4 -joint connector with three joints terminated by $50 \Omega$ terminators in a frequency window of $3.5-7.5 \mathrm{GHz}$ (see the inset in Fig. 1).

In order to investigate the distribution $P(R)$ of the reflection coefficient $R$ and the distributions of imaginary and real parts of the $K$ matrix we measured the scattering matrix $S_{\mathrm{s}}$ for 181 different realizations of tetrahedral microwave graphs having a microwave (SMA) attenuator in one of the bonds. For each graph realization, which was obtained either by the replacement of the bonds or putting an attenuator to a different bond, the scattering matrix $S$ was measured in 1601 equally spaced steps. The total optical lengths of the microwave graphs, including joints and the attenuator, was $196.2 \mathrm{~cm}$ when a $3 \mathrm{~dB}$, or $6 \mathrm{~dB}$ attenuator was used, whereas it was $197.4 \mathrm{~cm}$ when the $10 \mathrm{~dB}$ attenuator was used. To avoid degeneracy of eigenvalues in the graphs, we chose optical lengths for the bonds that were not simply commensurable.

Figure 2 shows the modulus $\left|S_{\mathrm{s}}\right|$ (part (a)) and the phase $\theta$ (part (b)) of the scattering matrix $S_{\mathrm{s}}$ of a tetrahedral graph with $\gamma=4.05$ (results were obtained with the use of a $3 \mathrm{~dB}$ attenuator) in a frequency range of $5.2-6.2 \mathrm{GHz}$.
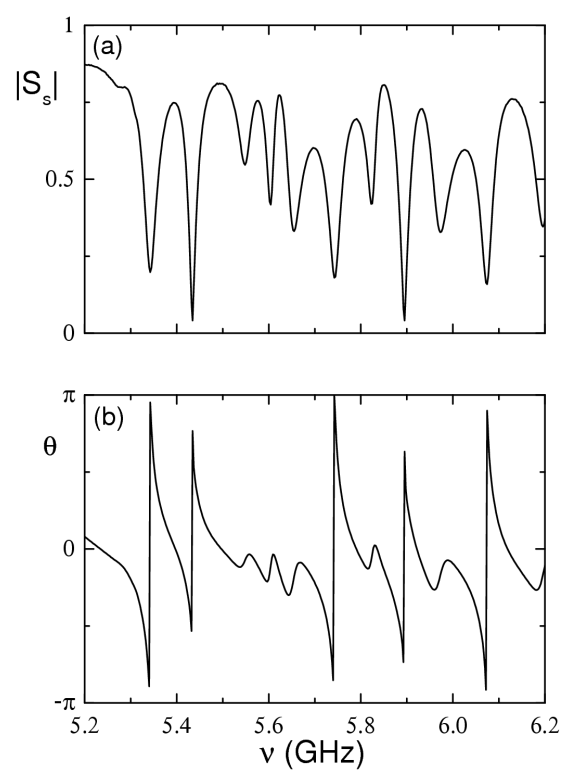

Fig. 2. Parts (a) and (b) show, respectively, the modulus $\left|S_{\mathrm{s}}\right|$ and the phase $\theta$ of the scattering matrix $S_{\mathrm{s}}$ measured for the graph (see Fig. 1) with absorption parameter $\gamma=4.05$ (see the text) over a frequency range of $5.2-6.2 \mathrm{GHz}$ and with the use of a $3 \mathrm{~dB}$ attenuator. The total optical length of the graph was equal to $196.2 \mathrm{~cm}$.

For systems with time reversal symmetry $(\beta=1)$, the explicit analytic expression for the distribution of the reflection coefficient $R$ is given by [25]: 


$$
P(R)=\frac{2}{(1-R)^{2}} P_{0}\left(\frac{1+R}{1-R}\right) .
$$

The probability distribution

$$
P_{0}(x)=-\frac{\mathrm{d} W(x)}{\mathrm{d} x}
$$

where the integrated probability distribution $W(x)$ [25] is given by the following formula:

$$
\begin{aligned}
& W(x)=\frac{x+1}{4 \pi}\left[f_{1}(w) g_{2}(w)+f_{2}(w) g_{1}(w)\right. \\
& \left.+h_{1}(w) j_{2}(w)+h_{2}(w) j_{1}(w)\right]_{w=(x-1) / 2},
\end{aligned}
$$

with the auxiliary functions defined as follows:

$$
\begin{aligned}
& f_{1}(w)=\int_{w}^{\infty} \mathrm{d} t \frac{\sqrt{t|t-w|} \mathrm{e}^{-\gamma t / 2}}{(1+t)^{3 / 2}}\left[1-\mathrm{e}^{-\gamma}+t^{-1}\right] \\
& g_{1}(w)=\int_{w}^{\infty} \mathrm{d} t \frac{\mathrm{e}^{-\gamma t / 2}}{\sqrt{t|t-w|}(1+t)^{3 / 2}}, \\
& h_{1}(w)=\int_{w}^{\infty} \mathrm{d} t \frac{\sqrt{|t-w|} \mathrm{e}^{-\gamma t / 2}}{\sqrt{t(1+t)}}\left[\gamma+\left(1-\mathrm{e}^{-\gamma}\right)(\gamma t-2)\right] \\
& j_{1}(w)=\int_{w}^{\infty} \mathrm{d} t \frac{\mathrm{e}^{-\gamma t / 2}}{\sqrt{t|t-w|} \sqrt{1+t}}, \\
& f_{2}(w)=\int_{0}^{w} \mathrm{~d} t \frac{\sqrt{t|t-w|} \mathrm{e}^{-\gamma t / 2}}{(1+t)^{3 / 2}}\left[1-\mathrm{e}^{-\gamma}+t^{-1}\right], \\
& g_{2}(w)=\int_{0}^{w} \mathrm{~d} t \frac{\mathrm{e}^{-\gamma t / 2}}{\sqrt{t|t-w|}(1+t)^{3 / 2}}, \\
& h_{2}(w)=\int_{0}^{w} \mathrm{~d} t \frac{\sqrt{|t-w|} \mathrm{e}^{-\gamma t / 2}}{\sqrt{t(1+t)}}\left[\gamma+\left(1-\mathrm{e}^{-\gamma}\right)(\gamma t-2)\right], \\
& j_{2}(w)=\int_{0}^{w} \mathrm{~d} t \frac{\mathrm{e}^{-\gamma t / 2}}{\sqrt{t|t-w|} \sqrt{1+t}} .
\end{aligned}
$$

The distributions of the imaginary and the real parts $P(v)$ and $P(u)$ of the $K$ matrix [24] are given by the following formulae:

and

$$
P(v)=\frac{\sqrt{2}}{\pi v^{3 / 2}} \int_{0}^{\infty} \mathrm{d} q P_{0}\left[q^{2}+\frac{1}{2}\left(v+\frac{1}{v}\right)\right],
$$

$$
P(u)=\frac{1}{2 \pi \sqrt{u^{2}+1}} \int_{0}^{\infty} \mathrm{d} q P_{0}\left[\frac{\sqrt{u^{2}+1}}{2}\left(q+\frac{1}{q}\right)\right],
$$


where $-v=\operatorname{Im} K<0$ and $u=\operatorname{Re} K$ are, respectively, the imaginary and real parts of the $K$ matrix.

Figure 3 shows experimental distributions $P(R)$ (symbols) of the reflection coefficient $R$ for three mean values of the parameter $\bar{\gamma}$, viz., 4.11, 5.14, and 7.64. The distribution for $\bar{\gamma}=4.11$ is obtained by averaging over 67 realizations of microwave graphs having $\gamma$ within a window of $[3.72,4.66]$. The distribution for $\bar{\gamma}=5.14$ is obtained by averaging over 61 realizations of microwave graphs having $\gamma$ within a window of $[4.66,5.62]$. The distribution for $\bar{\gamma}=7.64$ is obtained by averaging over 53 realizations of microwave graphs having $\gamma$ within a window of $[6.60,8.59]$. We estimated the experimental values of the $\gamma$ parameter by adjusting the theoretical mean reflection coefficient $\langle R\rangle_{\text {th }}$ to the experimental one $\langle R\rangle=\left\langle S S^{\dagger}\right\rangle$, where

$$
\langle R\rangle_{\mathrm{th}}=\int_{0}^{1} \mathrm{~d} R R P(R) .
$$

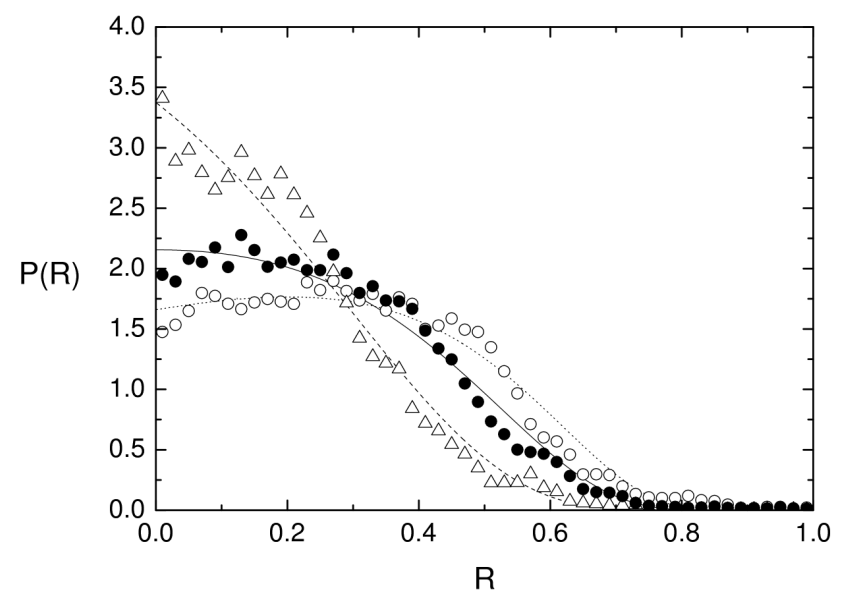

Fig. 3. Experimental distribution $P(R)$ of the reflection coefficient at different values of the mean absorption parameter: $\bar{\gamma}=4.11$ (open circles), $\bar{\gamma}=5.14$ (full circles), and $\bar{\gamma}=7.64$ (open triangles), respectively, calculated using the impedance approach $[26,27]$. Each corresponding theoretical distribution $P(R)$ evaluated from Eq. (4) is also shown: $\gamma=4.11$ (dotted line), $\gamma=5.14$ (solid line), and $\gamma=7.64$ (dashed line), respectively.

Figure 3 also presents the comparison of the experimental distributions $P(R)$ with the corresponding numerical distributions (lines) evaluated from Eq. (17). We see good overall agreement of the experimental distributions $P(R)$ with their theoretical counterparts. However some discrepancies are visible.

It is instructive to point out that the application in Eq. (17) of the often used approximated interpolation formula, valid for intermediate and strong absorption [32], for the distribution $P(R)$ : 


$$
P(R)=N_{\beta} \frac{\exp \left(-\frac{\alpha}{1-R}\right)}{(1-R)^{2+\beta / 2}}\left[A \alpha^{\beta / 2}+B(1-R)^{\beta / 2}\right]
$$

where $\alpha=\gamma \beta / 2, A=\mathrm{e}^{\alpha}-1, B=1+\alpha-\mathrm{e}^{\alpha}$, the normalization constant is $N_{\beta}=\alpha\left(A \Gamma(\beta / 2+1, \alpha)+B \mathrm{e}^{-\alpha}\right)^{-1}$ and $\Gamma(x, \alpha)=\int_{\alpha}^{\infty} \mathrm{d} t t^{x-1} \mathrm{e}^{-t}$ is the upper incomplete gamma function, yielded the following values of the parameter $\bar{\gamma}$ : 4.42, $5.50,8.05$. These values are slightly higher than the corresponding results obtained using the exact formula for $P(R)$ distribution.

The distributions of the imaginary and real parts of Wigner's reaction matrix $P(v)$ and $P(u)$ for three mean values of the parameter $\bar{\gamma}$, viz., 4.11, 5.14, and 7.64 are shown in Fig. 4 and Fig. 5, respectively. The experimental results are compared with the exact theoretical ones calculated from Eq. (15) and Eq. (16), respectively. We see that the experimental distributions $P(v)$ in Fig. 4 are generally in good agreement with their theoretical counterparts. However, some discrepancies are seen close to maximum values of the distributions $P(v)$, where the experimental results are slightly higher than the theoretical ones.

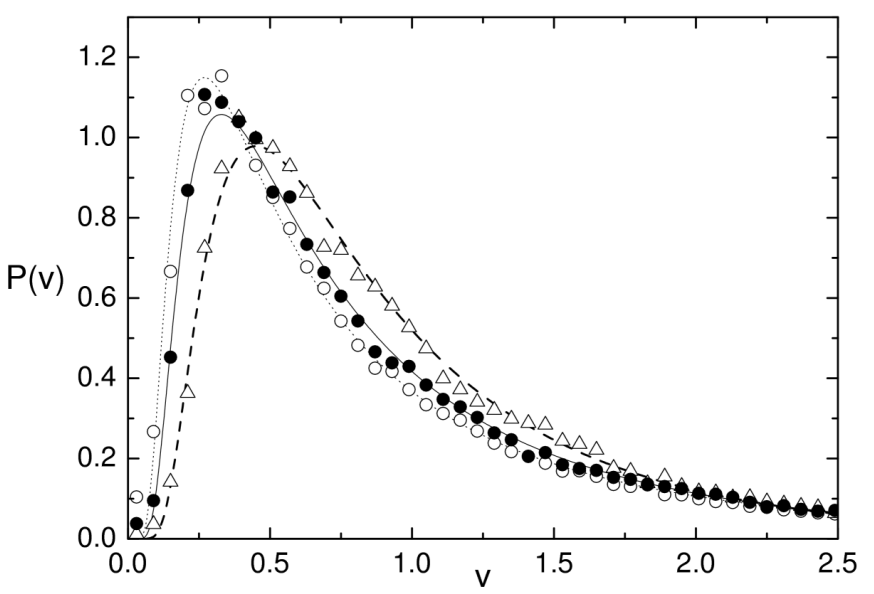

Fig. 4. Experimental distribution $P(v)$ of the imaginary part of the $K$ matrix at different values of the mean absorption parameter: $\bar{\gamma}=4.11$ (open circles), $\bar{\gamma}=5.14$ (full circles), and $\bar{\gamma}=7.64$ (open triangles), respectively, calculated using the impedance approach. Each corresponding theoretical distribution $P(v)$ evaluated from Eq. (15) is also shown: $\gamma=4.11$ (dotted line), $\gamma=5.14$ (solid line), and $\gamma=7.64$ (dashed line), respectively.

The distribution $P(u)$ of the real part of Wigner's reaction matrix has no direct physical meaning, however we may use these measurements for an important and natural consistency check on our determination of $\gamma$. Also in the case of the real part of Wigner's reaction matrix $P(u)$ presented in Fig. 5 the overall agreement between the experimental and exact theoretical results is good. However, for all three cases the middle $(-0.25<u<0.25)$ of the experimental distribution is 




Fig. 5. Experimental distribution $P(u)$ of the real part of the $K$ matrix at different values of the mean absorption parameter: $\bar{\gamma}=4.11$ (open circles), $\bar{\gamma}=5.14$ (full circles), and $\bar{\gamma}=7.64$ (open triangles), respectively, calculated using the impedance approach. Each corresponding theoretical distribution $P(u)$ evaluated from Eq. (16) is also shown: $\gamma=4.11$ (dotted line), $\gamma=5.14$ (solid line), and $\gamma=7.64$ (dashed line), respectively.

slightly higher than its theoretical counterpart. According to the definition of the $K$ matrix (see Eq. (1)), such behavior of the experimental distribution $P(u)$ suggests an excess of small values of $|\operatorname{Im} S|$. We do not yet know the origin of this excess.

\section{Conclusion}

In summary, using the impedance approach we have measured the distribution $P(R)$ of the reflection coefficient $R$ and the distributions $P(v)$ and $P(u)$ of imaginary and real parts of Wigner's reaction matrix for irregular microwave graphs consisting of SMA cables, joints, and attenuators. The application of attenuators allowed us to vary absorption in the graphs in a controlled, quantitative way. The measurements were done for a single-channel antenna in the case of time reversal symmetry $(\beta=1)$. The experimental results for $P(R), P(v)$ and $P(u)$ obtained at the same three values of the mean parameter $\bar{\gamma}$ are in good overall agreement with the recent exact theoretical predictions [25].

\section{Acknowledgments}

This work was partially supported by the Ministry of Science and Higher Education grant No. N202 099 31/0746. 


\section{References}

[1] L. Pauling, J. Chem. Phys. 4, 673 (1936).

[2] H. Kuhn, Helv. Chim. Acta 31, 1441 (1948).

[3] C. Flesia, R. Johnston, H. Kunz, Europhys. Lett. 3, 497 (1987).

[4] R. Mitra, S.W. Lee, Analytical Techniques in the Theory of Guided Waves, Macmillan, New York 1971.

[5] Y. Imry, Introduction to Mesoscopic Systems, Oxford, New York 1996.

[6] D. Kowal, U. Sivan, O. Entin-Wohlman, Y. Imry, Phys. Rev. B 42, 9009 (1990).

[7] E.L. Ivchenko, A.A. Kiselev, JETP Lett. 67, 43 (1998).

[8] J.A. Sanchez-Gil, V. Freilikher, I. Yurkevich, A.A. Maradudin, Phys. Rev. Lett. 80, 948 (1998).

[9] Y. Avishai, J.M. Luck, Phys. Rev. B 45, 1074 (1992).

[10] T. Nakayama, K. Yakubo, R.L. Orbach, Rev. Mod. Phys. 66, 381 (1994).

[11] T. Kottos, U. Smilansky, Ann. Phys. 274, 76 (1999).

[12] T. Kottos, Acta Phys. Pol. A 109, 7 (2006).

[13] P. Pakoński, G. Tanner, K. Życzkowski, J. Stat. Phys. 111, 1331 (2003).

[14] P. Pakoński, K. Życzkowski, M. Kuś, J. Phys. A 34, 9303 (2001).

[15] G. Tanner, J. Phys. A 33, 3567 (2000).

[16] T. Kottos, H. Schanz, Physica E 9, 523 (2003).

[17] T. Kottos, U. Smilansky, J. Phys. A 36, 3501 (2003).

[18] F. Barra, P. Gaspard, J. Stat. Phys. 101, 283 (2000).

[19] R. Blümel, Yu. Dabaghian, R.V. Jensen, Phys. Rev. Lett. 88, 044101 (2002).

[20] O. Hul, Sz. Bauch, P. Pakoński, N. Savytskyy, K. Życzkowski, L. Sirko, Phys. Rev. E 69, 056205 (2004).

[21] O. Hul, O. Tymoshchuk, Sz. Bauch, P.M. Koch, L. Sirko, J. Phys. A 38, 10489 (2005)

[22] G. Akguc, L.E. Reichl, Phys. Rev. E 64, 056221 (2001).

[23] Y.V. Fyodorov, D.V. Savin, JETP Lett. 80, 725 (2004).

[24] M.L. Mehta, Random Matrices, Academic Press, New York 1991.

[25] D.V. Savin, H.-J. Sommers, Y.V. Fyodorov, JETP Lett. 82, 544 (2005).

[26] S. Hemmady, X. Zheng, E. Ott, T.M. Antonsen, S.M. Anlage, Phys. Rev. Lett. 94, 014102 (2005).

[27] S. Hemmady, X. Zheng, T.M. Antonsen Jr., E. Ott, S.M. Anlage, Acta Phys. Pol. A 109, 65 (2006).

[28] G. López, P.A. Mello, T.H. Seligman, Z. Phys. A 302, 351 (1981).

[29] E. Doron, U. Smilansky, Nucl. Phys. A 545, 455 (1992).

[30] P.W. Brouwer, Phys. Rev. B 51, 16878 (1995).

[31] D.V. Savin, Y.V. Fyodorov, H.-J. Sommers, Phys. Rev. E 63, 035202 (2001).

[32] U. Kuhl, M. Martinez-Mares, R.A. Méndez-Sánchez, H.-J. Stöckmann, Phys. Rev. Lett. 94, 144101 (2005). 
[33] E. Kogan, P.A. Mello, H. Liqun, Phys. Rev. E 61, R17 (2000).

[34] R.A. Méndez-Sánchez, U. Kuhl, M. Barth, C.V. Lewenkopf, H.-J. Stöckmann, Phys. Rev. Lett. 91, 174102 (2003).

[35] C.W.J. Beenakker, P.W. Brouwer, Physica E 9, 463 (2001).

[36] D.S. Jones, Theory of Electromagnetism, Pergamon Press, Oxford 1964, p. 254. 